\section{P3.429 DOES SEXUAL BEHAVIOUR IN MEN WHO HAVE SEX WITH MEN (MSM) CORRELATE WITH THEIR PERCEIVED RISK OF HIV TRANSMISSION?}

doi:10.1136/sextrans-2013-051184.0880

A Baugh, M Pammi. Nottingham University Hospital NHS Trust, Nottingham, UK

Background MSM account for an increasing number of new HIV diagnoses in the UK, with $23 \%$ having recently acquired infections. This is largely due to high risk sexual behaviour and lower risk perception. To explore this, we evaluated the sexual behaviour of our MSM cohort and correlated this to their perceived risk of HIV acquisition. Methods HIV negative MSM attending a large sexual health centre were invited to complete an anonymous questionnaire. Information regarding demographics, sexual behaviour in terms of partner number, condom use, type of sexual activity, awareness of partner's HIV status etc was collected. A risk score was calculated and then correlated with their perceived risk of HIV.

Results We present preliminary data on 64 MSM. 78\% of respondents were White British, 63\% aged between 16 and 35 years. 52\% of MSM had 2-5 partners, $19 \% 5-10$ and $8 \%$ over 10 partners. Only $42 \%$ of MSM report consistent use of condoms for receptive anal sex. A large proportion reported using alcohol before sex; only $11 \%$ reported drug use. $65 \%$ reported unprotected anal sex with $0-1$ partners and $32 \%$ with between $2-5$ partners. Only $15 \%$ always discussed HIV status before sex, but more (38\%) discussed condom use. Perceived risk of HIV: $34 \%$ thought it was low, $59 \%$ moderate and only $7 \%$ perceived themselves at high risk. Actual risk of HIV: According to the weighted scores $56 \%$ had low risk, $42 \%$ moderate and $2 \%$ high risk of HIV acquisition. Correlation: There is concordance between actual and perceived risk in 55\% of respondents. Majority who had low risk perceived themselves as having moderate or high risk of acquisition.

Conclusion Evaluating MSM at high risk of HIV acquisition will help identify who would benefit most from behavioural intervention. Full report and statistics will be provided for the conference.

\section{P3.430* DOXYCYCLINE PROPHYLAXIS FOR SYPHILIS IN A PERSISTENTLY HIGH RISK HIV INFECTED POPULATION}

doi:10.1136/sextrans-2013-051184.0881

R. K. Bolann ${ }^{1,2}$, M. Beymer ${ }^{1,3}$, J. D. Klausner ${ }^{3}$, R. Flynn', A. Leibowitz ${ }^{3}$. 'L.A. Gay \& Lesbian Center, Los Angeles, CA, United States, 2University of Southern California Department of Family Medicine, Keck School of Medicine, Los Angeles, CA, United States, ${ }^{3}$ University of California Los Angeles, Los Angeles, CA, United States

Background Syphilis infections increase spread of HIV. This pilot study investigated the feasibility of conducting a large, randomised trial to determine whether prophylactic doxycycline can reduce incident syphilis among HIV + men who have sex with men having two or more syphilis infections since HIV diagnosis.

Methods Thirty subjects from the L.A. Gay \& Lesbian Center were randomised to doxycycline $100 \mathrm{mg}$ daily (Doxy) or Contingency Management (CM). Behavioral and biomedical measurements were taken at baseline, 12, 24, 36 and 48 weeks. Binomial Random Effects Logistic Regression Models assessed changes in behavioural measurements and analysed STI contraction at followup visits. Fisher Exact Tests compared dropout rates. Given small sample size, an alpha level of 0.1 was chosen.

Results Study retention did not differ significantly between groups: 12 and 11 subjects in the Doxy and CM groups, respectively, completed the 48 week study (Fisher Exact p-value $=1.0$ ). On intent-to-treat basis 12 (80\%), 11 (73.3\%) and 11 (73.3\%) subjects had detectable doxycycline levels at 12, 24, and 36 weeks, respectively. New STIs for Doxy vs. CM groups during 9 month on-drug study portion were: syphilis 2 vs. 6 , Neisseria gonorrhoea
1 vs. 2, Chlamydia trachomatis 1 vs.3. CM subjects reported more condom use than Doxy subjects $(p=0.09)$, however CM group subjects had 3.47 greater odds ( $p$-value $=0.037$; CI: $1.09-11.03$ ) for a new STI compared to Doxy group subjects. Behavioral outcomes for drug use $(p=0.91)$, anonymous partners $(p=0.45)$, and number of regular partners $(p=0.14)$ were not significantly different.

Conclusion No safety concerns were associated with daily administration of doxycycline. High retention and drug adherence rates suggest this high risk population will participate in an effectiveness study using doxycycline to reduce syphilis incidence. Efficacy of doxycycline in decreasing incidence of STIs in this pilot study further justifies a definitive study.

\section{P3.431 IMPACT OF SOCIAL MARKETING TO PROMOTE AWARENESS OF "EARLY" HIV TESTING IN ADDITION TO POOLED NUCLEIC ACID AMPLIFICATION TESTING IN MEN WHO HAVE SEX WITH MEN IN VANCOUVER, BRITISH COLUMBIA}

doi:10.1136/sextrans-2013-051184.0882

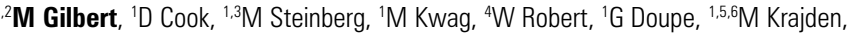
${ }^{2} \mathrm{M}$ Rekart. 'British Columbia Centre for Disease Control, Vancouver, BC, Canada,

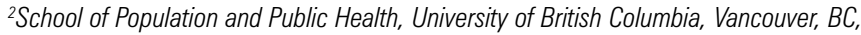
Canada; ${ }^{3}$ Faculty of Health Sciences, Simon Fraser University, Burnaby, BC, Canada; ${ }^{4}$ Health Initiative for Men, Vancouver, BC, Canada; ${ }^{5}$ Faculty of Medicine, University of British Columbia, Vancouver, BC, Canada; ${ }^{6} B C$ Public Health Microbiology Reference Laboratory, Vancouver, BC, Canada

Background The contribution of acute HIV infection (AHI) to transmission is widely recognised. Increasing AHI diagnosis capacity can enhance HIV prevention through subsequent behaviour change or intervention. We examined the impact of targeted pooled NAAT and social marketing to increase AHI diagnosis among men who have sex with men (MSM) in Vancouver, British Columbia.

Methods We implemented pooled NAAT following negative $3^{\text {rd }}$ generation EIA testing for males $\geq 19$ years in six clinics accessed by MSM, accompanied by two social marketing campaigns developed in partnership with a community-based gay men's health organisation (campaigns emphasised availability of "early" testing for AHI and promoted early testing following potential exposure or new relationship). We compared test volume and diagnosis rates for pre(April 2006-March 2009) and post-pooling (April 2009-March 2012) periods, and calculated diagnostic rate and yield, RNA copy number, time to results and cost per diagnosis.

Results Post-pooling, the AHI diagnosis rate increased from 1.0 to 1.84 per 1,000 tests; quarterly AHI diagnosis rates increased significantly. Of 217 new HIV diagnoses post-pooling, 54 (24.9\%) were AHI (25 detected by pooled NAAT only) for an increased diagnostic yield of $11.5 \%$. AHI detected by pooled NAAT had higher median RNA copies $\left(7.77 \times 10^{5}\right.$ copies $\left./ \mathrm{mL}\right)$ and similar time to result (median 7 days) compared to AHI detected through $3^{\text {rd }}$ generation EIA $\left(4.96 \times 10^{5}\right.$ copies $/ \mathrm{mL}$; median 6 days). The incremental cost per AHI diagnosis through pooled NAAT was $\$ 9,124 \mathrm{CDN}$

Conclusions Few studies have assessed the contribution of social marketing in enhancing capacity for AHI diagnosis. Our study suggests that targeted implementation of pooled NAAT at clinics accessed by MSM accompanied by local social marketing campaigns is effective at increasing AHI diagnoses, and is likely cost-saving.

\section{P3.432 TRENDS IN RISK BEHAVIOR AND HIV AND SYPHILIS INFECTION AMONG MSM WHO ATTEND IN STI CLINICS IN GUATEMALA}

doi:10.1136/sextrans-2013-051184.0883

S Morales Miranda, C Galindo Arandi, F Arana, I Loya. Universidad del Valle de Guatemala, Guatemala, Guatemala 